\title{
CURCUMINA E ANÁLAGOS SINTÉTICOS: POSSÍVEIS SUPLEMENTOS ALIMENTARES PARA ATUAÇÃO EM ALTERAÇÕES OXIDATIVAS DE DIABÉTICOS
}

\author{
Marina Ferrara de Resende ${ }^{1}$ \\ Renata Barbosa de Oliveira ${ }^{2}$ \\ Renata Adriana Labanca ${ }^{3}$
}

\begin{abstract}
Resumo: Espécies reativas são continuamente produzidas pelo organismo, mas quando excedem a defesa antioxidante geram uma condição conhecida por estresse oxidativo, relacionado a várias doenças crônicodegenerativas como o diabetes mellitus (DM). O DM é caracterizado por hiperglicemia crônica, que resulta em desequilíbrio metabólico e dano tecidual. Assim, o aparecimento dessa patologia está altamente relacionado aos hábitos de vida e alimentares da população, sendo de grande interesse a busca por suplementos alimentares que possam também atuar evitando ou diminuindo alterações oxidativas em indivíduos diabéticos. Nesse contexto, suplementos alimentares constituídos por antioxidantes representam uma defesa muito eficiente de combate ao estresse oxidativo e, consequentemente, ao diabetes e suas complicações. Dessa maneira, destaca-se então, a conhecida ação antioxidante apresentada pela curcumina e também seus análogos sintéticos, sugerindo esses compostos para uso como promissores suplementos alimentares de forma a atuar como protetores do organismo.

Palavras-chaves: curcumina, suplementos alimentares, diabetes
\end{abstract}

\section{CURCUMINA AND SYNTHETICAL ANALOGUES: POSSIBLE FOOD SUPPLEMENTS FOR ACTION IN DIABETIC OXIDATIVE CHANGES}

\begin{abstract}
Reactive species are continually being produced by the body, but when they exceed the antioxidant defence capacity generate a condition known as oxidative stress, being related to several chronic diseases, such as diabetes mellitus (DM). Such disease is characterized by chronic hyperglycemia, resulting in metabolic imbalance and tissue damage. Thus, the appearance of such disease is highly correlated to population's lifestyle and dietary habits, being of great interest the search for dietary supplements that may also act by preventing or decreasing oxidative changes in diabetic subjects. In this context, dietary supplements consisting of antioxidants are a very efficient protection to fight oxidative stress and, hence, diabetes and its complications. In this way, it stands out then, the well-known antioxidant activity shown by curcumin, as well as its synthetic analogues, has suggested these compounds to be used as promising dietary supplements, that would act as body protectors.
\end{abstract}

Keywords: curcumin, dietary supplements, diabetes

\section{CURCUMINA Y SINTÉTICO ANÁLOGOS: LOS SUPLEMENTOS DIETÉTICOS POSIBLES PARA LA ACCIÓN EN CAMBIOS OXIDATIVO DE DIABETES}

\begin{abstract}
Resumen: Especies reactivas se producen continuamente por el cuerpo, pero cuando exceden la defensa antioxidante generar una condición conocida como estrés oxidativo, relacionado con varias enfermedades degenerativas crónicas tales como diabetes mellitus (DM). Lo DM se caracteriza por la hiperglucemia crónica, resultando en desequilibrio metabólico y daño tisular. Por lo tanto, la aparición de esta enfermedad es relacionado con el estilo de vida y la alimentación de la población, siendo de gran interés para la búsqueda de los suplementos dietéticos que pueden actuar también mediante la prevención o la reducción de las alteraciones oxidativas. En este contexto, los suplementos alimentarios consistentes en antioxidantes son una protección muy eficaz para combatir el estrés oxidativo y, en consecuencia, la diabetes y sus complicaciones. De esta manera, se destaca luego, la acción antioxidante bien conocido presentado por la curcumina y también sus análogos sintéticos, lo que sugiere que estos compuestos para uso como suplementos dietéticos prometedores para actuar como guardias de cuerpo.

Palabras clave: curcumina, suplementos dietéticos, diabetes
\end{abstract}

\footnotetext{
${ }^{1}$ Mestre do Programa de Pós Graduação em Ciência de Alimentos da Faculdade de Farmácia da Universidade Federal de Minas Gerais.

${ }^{2}$ Professora do Departamento de Alimentos da Faculdade de Farmácia da Universidade Federal de Minas Gerais.

${ }^{3}$ Professora do Departamento de Ciêcias Farmacêuticas da Faculdade de Farmácia da Universidade Federal de Minas Gerais.
} 


\section{Introdução}

O organismo humano, por meio de suas funções bioquímicas normais, produz continuamente espécies reativas, contra as quais existe um sistema de defesa antioxidante. Porém, quando há um desequilíbrio nessa relação, instala-se uma condição conhecida por estresse oxidativo, onde as espécies reativas geram danos cumulativos à biomoléculas, o que pode gerar várias doenças crônico-degenerativas, entre elas o diabetes mellitus (SILVA et al., 2011; CAROCHO; FERREIRA, 2013; ZHAO et al., 2014).

Diabetes mellitus é uma doença metabólica caracterizada por hiperglicemia crônica, que afeta o metabolismo associado ao estresse oxidativo, levando a sérios problemas de saúde. O aumento dessa doença na população mundial, atribuído não somente ao crescente número de idosos, mas principalmente ao estilo de vida e de alimentação atuais, faz com que essa doença tenha grande impacto nos gastos com a saúde. Desta forma, torna-se importante controlar seu agravamento a fim de diminuir o sofrimento dos pacientes e conter custos sociais (HENRIQUES et al., 2010; WHO, 2015).

Dessa maneira, tem crescido nos últimos anos o interesse por substâncias antioxidantes, naturalmente presente nos alimentos ou de origem sintética, que possam ser utilizadas como suplementos alimentares, sendo eficazes na prevenção do surgimento ou agravamento do diabetes, com o mínimo de efeitos colaterais. (HUSSEIN; ABU-ZINADAH, 2010; PRABHAKAR et al., 2013; JEENGER et al. 2014).

Nesse contexto, destaca-se a curcumina, principal ativo extraído dos rizomas da cúrcuma ou açafrão (Curcuma longa), sendo esta planta tradicionalmente utilizada na culinária e na medicina chinesa e indiana e também usada no Brasil como tempero (HATCHER et al., 2008; PIRES et al., 2013). A curcumina é amplamente estudada por possuir grande poder antioxidante e excelente perfil de segurança e pode ser utilizada como suplemento alimentar para atuar na proteção às biomoléculas, associada à melhora no tratamento do diabetes e de suas complicações. (ANAND et al., 2008; VOLP et al., 2009; HONORATO et al., 2013; PRASAD et al., 2014). O artigo teve como objetivo verificar as propriedades funcionais da cúrcuma, um derivado do rizoma seco da Curcuma longa, administrado como suplementação nutricional associado principalmente ao diabetes mellitus.

\section{Metodologia}


Para atender ao objetivo nomeado, foi feito um estudo exploratório, descritivo e documental, mediante levantamento bibliográfico dos artigos disponíveis nos bancos de dados LILACS, MEDLINE, BDENF e SciELO no período de 1993 a 2016, produzidos no Brasil e no exterior. Para tanto, usaram-se como descritores os termos diabetes mellitus, análogos sintéticos, suplementos alimentares e alterações oxidativas, sendo selecionados os artigos originais, que estavam disponíveis na íntegra. A coleta de dados ocorreu em junho/2016, quando foram obtidos, através dos descritores, mais de cem artigos, os quais foram lidos e criticamente analisados. Em seguida, selecionaram-se aqueles que abordavam diretamente o assunto. Ao final, restaram setenta artigos os quais foram sintetizados em informações nesse texto de revisão.

\section{Radicais Livres, Estresse Oxidativo e Diabetes}

Reações bioquímicas que ocorrem nas células e organelas do corpo humano são inerentes à manutenção da vida. O consumo de oxigênio envolvido no processo de respiração, característico do crescimento celular, além de outros eventos fisiológicos normais, conduzem à geração de moléculas altamente instáveis, conhecidas como espécies reativas (ERs). (FERREIRA \& MATSUBARA, 1997; CAROCHO \& FERREIRA, 2013).

As ERs incluem espécies radicalares (radicais livres) e não radicalares. Os radicais livres (RLs) são átomos, moléculas ou íons com um ou mais elétrons desemparelhados, altamente instáveis e reativos. As principais fontes de RLs são as organelas citoplasmáticas que metabolizam o oxigênio e o nitrogênio, gerando grande quantidade de metabólitos. (FERREIRA \& MATSUBARA, 1997; BARBOSA et al., 2008; CAROCHO \& FERREIRA, 2013).

Uma série de ERs de oxigênio (EROs) e nitrogênio (ERNs) são continuamente produzidas pelo organismo, sendo que muitos desses processos formam ERs com efeitos benéficos e importantes, como geração de ATP por meio da cadeia transportadora de elétrons, fertilização de óvulos, ativação de genes e participação em mecanismos de defesa durante processos infecciosos. (FERREIRA \& MATSUBARA, 1997; BARBOSA et al., 2010).

Porém, quando a quantidade dessas espécies está muito elevada e excede as defesas do organismo, resulta em uma condição conhecida por estresse oxidativo. ERs podem, por exemplo, iniciar facilmente a peroxidação lipídica de membranas celulares e causar danos cruciais às biomoléculas, tais como ácidos nucléicos, lipídeos, proteínas e carboidratos, podendo causar mutações, gerar apoptose de células saudáveis, envelhecimento precoce, alteração da função celular e aparecimento de doenças crônico-degenerativas. (AK \& GÜLÇIN, 2008; CHOI, 2009). 
Portanto, o estresse oxidativo pode ser caracterizado como um estado de desequilíbrio entre a produção de ERs e a defesa antioxidante, evidenciando a incapacidade de antioxidantes endógenos em neutralizar o dano oxidativo em alvos biológicos. (SILVA et al., 2011; LÓPEZALARCÓN\& DENICOLA, 2013).

A maioria das espécies reativas presentes no organismo humano são derivadas do oxigênio, formando as EROs que incluem, entre outros, radical superóxido $\left(\mathrm{O}_{2}{ }^{\bullet-}\right)$, radical hidroperoxila $\left(\mathrm{HO}_{2}{ }^{\circ}\right)$, radical hidroxila $\left({ }^{\circ} \mathrm{OH}\right)$, óxido nítrico $\left(\mathrm{NO}^{\circ}\right)$, e outras espécies não radicalares, como o peróxido de hidrogênio $\left(\mathrm{H}_{2} \mathrm{O}_{2}\right)$, oxigênio singlete $\left({ }^{1} \mathrm{O}_{2}\right)$, ácido hipocloroso ( $\mathrm{HOCl})$ e peroxinitrito $\left(\mathrm{ONOO}^{-}\right)$. (BARBOSA et al., 2008; CAROCHO \& FERREIRA, 2013).

Como já mencionado, os RLs são produzidos como uma parte normal do metabolismo, porém fatores externos também podem ajudar a promover sua produção, como o consumo de álcool, cigarro e junk foods, poluentes ambientais, radiação, medicamentos, pesticidas, solventes industriais e ozônio (CAROCHO \& FERREIRA, 2013; YADAV et al. 2013). Em quantidades baixas ou moderadas, os RLs apresentam uma função biológica importante, porém são altamente reativos. (NIKI, 2010; CAROCHO \& FERREIRA, 2013).

Em relação às proteínas, existem três vias distintas que podem ser modificadas oxidativamente: modificação de um aminoácido específico, clivagem de peptídeo mediada por radical livre e formação de ligação cruzada devido à reação com produtos da peroxidação lipídica. Tais modificações podem levar à degradação, alteração da estrutura terciária e da funcionalidade e perda da atividade enzimática (KOHEN \& NYSKA, 2002; CAROCHO \& FERREIRA, 2013).

O padrão de danos induzidos por radicais livres no DNA pode ser descrito tanto quimicamente quanto estruturalmente da seguinte forma: modificação das bases nitrogenadas, deleções, produção de sítios sem base, mudança no quadro de leitura, quebras de fita, ligação cruzada de DNA e proteína e rearranjos cromossômicos. Uma importante reação que resulta em danos ao DNA é a produção de radical hidroxila através da reação de Fenton. Esse radical é conhecido por reagir com todos os componentes da molécula de DNA: as bases púricas e pirimídicas e o esqueleto desoxirribose-fosfato. (HALLIWELL \& CHIRICO, 1993; KOHEN \& NYSKA, 2002; BARBOSA et al., 2008; CAROCHO \& FERREIRA, 2013).

Em relação aos carboidratos, a formação de EROs durante a glicação precoce pode contribuir para danos glicoxidativos. Durante os estágios iniciais da glicosilação enzimática, a fragmentação do açúcar produz espécies de cadeia curta como glicoaldeído que não ciclizam e, por conseguinte, são propensas a auto-oxidação, formando o radical superóxido. O resultado da 
reação em cadeia propagada por este radical é a formação de compostos $\alpha$ e $\beta$-dicarbonílicos, que são mutagênicos bem conhecidos. (CAROCHO \& FERREIRA, 2013).

Quanto aos lipídeos, o ataque de um radical, principalmente ${ }^{\circ} \mathrm{OH}$, à cadeia lateral de um ácido graxo de membranas celulares, a fim de abstrair um átomo de hidrogênio de um carbono metilênico, dá início ao processo conhecido como peroxidação lipídica. Tal evento induz distúrbios em estruturas celulares, alteração da integridade, fluidez e permeabilidade de membranas, perda funcional de membranas, modificações em lipoproteínas de baixa densidade (LDL) para formas pró-aterogênicas e pró-inflamatórias, e formação de produtos como o malondialdeído (MDA), fatores estes que podem culminar em morte celular. Além disso, produtos da lipoperoxidação como os compostos carbonilados têm sido citados como mutagênicos, carcinogênicos e indutores de morte celular. (FERREIRA \& MATSUBARA, 1997; GROTTO et al., 2009; CAROCHO \& FERREIRA, 2013).

Estas reações supracitadas levam à produção de ERs, cujo excesso tem sido correlacionado a várias doenças graves como diabetes mellitus e suas complicações, conforme citado anteriormente. (DAI et al., 2009; CAROCHO \& FERREIRA, 2013; YADAV et al., 2013).

O diabetes mellitus (DM) é uma doença metabólica crônica não transmissível que tem se tornado um problema global devido ao aumento da incidência e prevalência dessa patologia que vem associada a sérias consequências para a saúde humana (MENG et al., 2013; WHO, 2015). Dados mostram que em 2012 essa doença foi a causa direta de 1,5 milhão de mortes, e a Organização Mundial de Saúde (OMS) estima que as mortes dobrem entre os anos de 2005 e 2030, sendo a sétima principal causa de morte em 2030 (WHO, 2015).

O Brasil é o oitavo país com maior prevalência dessa doença, que junto à hipertensão, é responsável pelo maior número de mortes. O DM é também a principal causa de internações por complicações relacionadas à doença cardiovascular, diálise por insuficiência renal crônica e amputações de membros inferiores. (HENRIQUES et al., 2010; PAHO, 2015).

O crescente aumento dessa doença é atribuído ao envelhecimento da população e, principalmente, ao estilo de vida atual caracterizado pelo sedentarismo e hábitos alimentares que predispõem ao acúmulo de gordura corporal. (HENRIQUES et al., 2010). É fato que complicações diabéticas sempre resultam no aumento da morbidade ou mortalidade entre os seres humanos, fazendo com que o DM seja uma das doenças crônicas de maior impacto nos gastos com saúde. (PEEYUSH et al., 2009; PAHO, 2015). 
O DM é caracterizado por insuficiência na secreção de insulina, hormônio produzido e secretado pelas células $\beta$ das ilhotas de Langerhans do pâncreas, ou por sensibilidade reduzida dos tecidos a esse hormônio (ou resistência periférica à insulina), ou ainda pela combinação dos dois fatores, resultando assim em hiperglicemia. (PRABHAKAR et al., 2013; NAJAFIAN, 2014; WHO, 2015). Tal distúrbio afeta o metabolismo de carboidratos, proteínas e lipídeos, predispondo os pacientes a sérios problemas de saúde, até mesmo a disfunção de múltiplos órgãos (PRABHAKAR et al., 2013; MENG et al., 2013), afetando células $\beta$-pancreáticas, fígado, rim, cérebro, coração e outros órgãos. (AGGARWAL; HARIKUMAR, 2009; REDDY et al., 2010).

Em curto prazo, o DM gera complicações como a cetoacidose diabética e sintomas como visão turva, poliúria, polidipsia, polifagia, cetonúria, poliastenia, glicosúria, proteinúria, elevação da ureia urinária e elevação plasmática nos níveis de determinadas enzimas hepáticas. A cetoacidose diabética ocorre pela grande mobilização de ácidos graxos do tecido adiposo (lipólise) seguida da oxidação desses ácidos graxos, disponibilizando moléculas de acetil-CoA que são substratos para a cetogênese hepática. Eventos de hipoglicemia são considerados um efeito colateral do tratamento do diabetes. (CHAMPE et al., 2006; WHO, 2015).

Em longo prazo, o DM pode acarretar complicações vasculares, como nefropatia, neuropatia e retinopatia, associadas às altas taxas de morbidade e mortalidade. (SOETIKNO et al., 2012; PRABHAKAR et al., 2013; SOETIKNO et al., 2013; JEENGER et al., 2014). Pacientes com DM também têm incidência aumentada de aterosclerose e doenças cardiovasculares, distúrbios endócrinos, alterações nas sensações somáticas que envolvem o córtex cerebral, cerebelo e tálamo levando ao déficit cognitivo e mudanças neurofisiológicas e estruturais. (SILVA et al., 2011; KUMAR et al., 2011; PRABHAKAR et al., 2013; WHO, 2015).

De acordo com a OMS, os tipos mais comuns de diabetes são o 1 e o 2, mas também existem o diabetes gestacional, desenvolvido durante a gravidez e outros tipos menos comuns gerados por fatores como síndromes genéticas, pancreatite, doenças como fibrose cística, exposição a certos fármacos, vírus e causas desconhecidas (WHO, 2015).

O DM tipo 1 é uma doença geralmente autoimune caracterizada por uma reação inflamatória local dentro e em torno das ilhotas pancreáticas que é seguida por destruição seletiva das células $\beta$-secretoras de insulina. Geralmente se desenvolve na infância e adolescência, e os pacientes necessitam de injeções de insulina por toda a vida para a sobrevivência. (ARORA et al., 2009; WHO, 2015). 
O DM tipo 2 é caracterizada por resistência periférica à insulina e secreção deficiente de insulina. Desenvolve-se geralmente na idade adulta e está relacionada com a obesidade, falta de atividade física e alimentação não saudável. Este é o tipo mais comum de diabetes (que representa $90 \%$ dos casos diabéticos em todo o mundo) e o tratamento pode envolver mudanças de estilo de vida e perda de peso por si só, medicação oral ou mesmo injeções de insulina. (ARORA et al., 2009; WHO, 2015).

Tem sido demonstrado que o estresse oxidativo desempenha um papel crítico nas complicações associadas ao DM. Essa forte associação se deve ao fato da hiperglicemia favorecer a formação de EROs, e algumas propostas para que isso ocorra são por meio da autooxidação da glicose, da formação de produtos finais de glicação avançada (AGEs), da via dos polióis e também das mudanças no conteúdo e atividade no sistema de defesas antioxidantes no tecido. Todos esses eventos podem levar ao dano oxidativo em membranas, rompimento de funções celulares e agravamento da peroxidação lipídica. (SILVA et al., 2011; MENG et al., 2013).

A produção aumentada de EROs durante a hiperglicemia diminui ainda o sistema de defesa antioxidante, que não consegue agir de maneira suficiente contra os radicais livres. Além disso, a oxidação de lipídios e de proteínas também está associada ao desenvolvimento das complicações do DM. (HUSSEIN; ABU-ZINADAH, 2010; SILVA et al., 2011; MENG et al., 2013).

Diante de tais considerações, é importante que os pacientes diabéticos estabeleçam um rigoroso controle da glicemia, uma vez que ainda não existe cura para essa doença. Assim, a intervenção farmacológica se faz necessária, porém os efeitos colaterais de diversos fármacos hipoglicemiantes já existentes e o desconforto do paciente quanto ao tratamento têm levado vários pesquisadores a buscar suplementos alimentares que atuem tanto na prevenção quanto na diminuição dos danos oxidativos causados pelo diabetes. (DEVCICH et al., 2007; ANAND et al., 2008; PRABHAKAR et al., 2013).

O envelhecimento da população, sedentarismo e hábitos alimentares ditos não-saudáveis, vem gerando um aumento e agravamento de doenças crônicas como o diabetes. Dessa maneira, é notória a necessidade de reduzir o surgimento de novos casos de diabetes e prevenir o aumento da morbidade e mortalidade no DM. Tal fato pode ser conseguido com mudanças no estilo de vida por meio da prática de atividade física regular e uma alimentação adequada e saudável, além de acompanhamento profissional (VIEIRA et al., 2011; GUIA, 2014). 
Assim, a alimentação saudável passou a ser um componente essencial para prevenção e tratamento do DM (HOROWITZ, 2004; DIABETES, 2015), visto que permite ao organismo manter o nível de glicose em equilíbrio, além de promover o bem-estar. (RECINE et al., 2001). Vários estudos têm orientado atitudes corretas para uma alimentação saudável, como a realização de cinco ou seis refeições diárias com variedade de frutas, legumes e verduras, diminuição de açúcar e gordura, ingestão preferencial de carboidratos integrais, abstenção de frituras e priorização de alimentos in natura e ricos em fibras. (HOROWITZ, 2004; GUIA, 2014; DIABETES, 2015).

De acordo com o Guia Alimentar para a População Brasileira (2014), publicado pelo Ministério da Saúde, frutas, legumes e verduras são excelentes fontes de vitaminas, minerais, fibras e antioxidantes, características que os tornam ideais para prevenção das doenças crônicas, como o diabetes. Porém, esses alimentos ainda são pouco consumidos devido ao estilo de vida da população, que têm demonstrado preferência por alimentos processados e ultraprocessados caracterizados por conter altos teores de carboidratos, gorduras e/ou aditivos, mas pobres em vitaminas e minerais. Uma opção para o consumidor é a complementação da dieta com nutrientes e outras substâncias que apresentem algum benefício para a saúde. Nesse contexto, ganham espaço e relevância os suplementos alimentares e nutricionais.

\section{Diabetes e Suplementos Alimentares}

No que se trata de um melhor controle glicêmico, diminuição da resistência à insulina e prevenção de complicações do diabetes, os suplementos alimentares já têm sido utilizados por anos como aliados para esses fins, junto a outras práticas de promoção da saúde como atividade física diária, alimentação saudável e acompanhamento profissional. (SHANE-MCWHORTER, 2012).

No Brasil a portaria ${ }^{\circ} 32$ de 13 de janeiro de 1998 da Secretaria Nacional de Vigilância Sanitária (ANVISA, 1998) aprovou o Regulamento Técnico para Suplementos Vitamínicos e/ou de Minerais e define-os como compostos vitamínicos e/ou de minerais que servem para complementar a dieta diária de uma pessoa saudável, em casos onde sua ingestão a partir da alimentação, seja insuficiente ou quando a dieta requerer suplementação. Segundo a regulamentação no país, os suplementos devem conter apenas vitaminas e/ou minerais com, no mínimo, 25\%, e no máximo, 100\% da Ingestão Diária Recomendada (IDR), na porção diária indicada pelo fabricante. Esses suplementos não podem substituir os alimentos, nem serem considerados como dieta exclusiva. Em muitos países, a categoria de suplementos alimentares 
abrange, além das vitaminas e minerais, outros nutrientes como fibras e aminoácidos, substâncias com efeito nutricional ou fisiológico, como os antioxidantes licopeno e luteína e/ou ácidos graxos como ômega 3 , entre outros.

Atualmente no Brasil, por questões regulatórias, produtos contendo outras substâncias além de vitaminas e minerais ainda não são identificados como suplementos alimentares no rótulo. (ANVISA, 1998).

A ampliação do conhecimento sobre os benefícios dos suplementos alimentares e nutricionais à saúde, as diversas finalidades desses suplementos e a constante inovação e avanço das tecnologias têm levado ao crescimento em ritmo acelerado do mercado de suplementos no Brasil e no mundo (SENADO, 2014; SILVA \& FERREIRA, 2014).

A legislação atual sobre o setor de suplementos alimentares e nutricionais é composta pelo decreto-Lei $\mathrm{n}^{\circ}$ 986, de 21 de outubro de 1969, por três leis vigentes desde a década de 1970 (Leis $n^{\circ} 5.991$, de 1973, 6.360, de 1976, e 6.437, de 1977) e pela portaria $n^{\circ} 32$ de 13 de janeiro de 1998. Além disso, a variedade comercializada no Brasil possui diferentes designações e classificações, com ausência de uma regulamentação específica para essa multiplicidade que vem surgindo. Assim, o cenário regulatório atual se mostra desatualizado, inseguro e confuso para os consumidores e até mesmo para os agentes de vigilância sanitária (ANVISA, 1998; SENADO, 2014).

Diante disso, o Projeto de Lei $\mathrm{n}^{\circ} 233$, de 2014, tramita pelo senado dispondo sobre os suplementos alimentares e nutricionais, com proposta de atualizar a regulação do setor para garantir a observância de padrões de qualidade, eficácia e segurança dos suplementos. De acordo com esse projeto, suplementos alimentares e nutricionais, internacionalmente conhecidos por food supplements ou dietary supplements, são definidos como produtos compostos por nutrientes, fitonutrientes, outras substâncias encontradas nos alimentos e compostos bioativos que se destinam a suplementar a dieta habitual ou suprir necessidades nutricionais, metabólicas ou fisiológicas (BRASIL, 2014).

Vários suplementos alimentares já vêm sendo utilizados por pacientes diabéticos como a canela, amora, coenzima Q10, hibisco, vinagre, curcumina ou cúrcuma entre outros (AGGARWAL; HARIKUMAR, 2009; SHANE-MCWHORTER, 2012; MENG et al., 2013). Com base em uma pesquisa com adultos diabéticos foi demonstrado que $67 \%$ dos pacientes estavam usando algum tipo de vitamina ou suplemento (GARROW; EGEDE, 2006). Uma revisão das histórias de medicação de 459 indivíduos com diabetes indicou ainda que 55\% usam algum tipo de suplemento em sua alimentação diária (ODEGARD, 2011). 
Sugerem-se que os motivos pelos quais os pacientes diabéticos optam por tomar suplementos seja o alto custo e o desejo de evitar efeitos adversos dos medicamentos convencionais, além da influência cultural e de amigos. Assim, é importante reconhecer a ação e o alcance desses produtos, sendo necessário, além do acompanhamento profissional e a definição da dose diária recomendada, uma regulamentação que garanta a qualidade e a segurança dos suplementos alimentares (SHANE-MCWHORTER, 2012).

\section{Antioxidantes e Curcumina, um Possível Suplemento Alimentar}

Durante o curso da evolução, uma rede de defesa muito eficiente tem se formado para combater o estresse oxidativo, sendo necessários diversos antioxidantes e enzimas com diferentes funções para formar o sistema de defesa in vivo. Os antioxidantes podem ser definidos como qualquer substância que atrasa, impede ou elimina os danos oxidativos a uma molécula alvo, gerando compostos com baixa ou nenhuma toxicidade. (MAGALHÃES et al., 2008; NIKI, 2010; CAROCHO; FERREIRA, 2013).

Além de localizados em diferentes lugares, intra e extracelulares, os antioxidantes podem agir de diversas formas, sendo sua capacidade e eficácia determinadas não somente por sua estrutura química, mas também por fatores como a reatividade química com os radicais livres, taxa de eliminação de radicais, destino de radical após neutralização (devendo este ser estável), interação com outros antioxidantes (podendo resultar em antioxidação sinérgica), concentração e mobilidade no meio, absorção, distribuição, retenção e metabolismo. (ANAND et al., 2008; NIKI, 2010; CAROCHO; FERREIRA, 2013).

O sistema antioxidante pode ser classificado em enzimático e não-enzimático. O primeiro caso está representado principalmente pelas enzimas antioxidantes endógenas superóxido dismutase (SOD), catalase (CAT) e glutationa peroxidase (GPx). Quanto ao sistema antioxidante não-enzimático, este se relaciona a um grupo de antioxidantes que podem ser agrupados em compostos produzidos in vivo (endógenos), como glutationa reduzida, ubiquinona e ácido úrico, e em compostos obtidos diretamente da dieta (exógenos), como vitaminas E, C, beta-caroteno e outros (VASCONCELOS et al., 2007; CAROCHO; FERREIRA, 2013).

Apesar da notável eficiência do sistema antioxidante endógeno, por si só ele não é suficiente quando o estresse oxidativo se instala, por isso, tornam-se necessários vários tipos de antioxidantes presentes na dieta para manter a concentração de radicais livres em baixos níveis (CAROCHO; FERREIRA, 2013). 
Os antioxidantes exógenos podem ser de origem natural ou sintética, sendo que muitos deles, contidos em alimentos, frutas, bebidas, especiarias e suplementos têm gerado grande interesse entre consumidores e pesquisadores devido à associação de seu uso frequente com a prevenção de desordens que envolvem o estresse oxidativo, como o diabetes. Algumas dessas substâncias naturais que merecem destaque são os polifenólicos, os carotenóides e as poliaminas (CHOI, 2009; NIKI, 2010; CAROCHO; FERREIRA, 2013; SHARMA et al., 2013). Já os sintéticos têm sido amplamente utilizados como aditivos alimentares para atuar na proteção contra a degradação oxidativa de alimentos, aumentando sua vida de prateleira. Com esse objetivo, os antioxidantes sintéticos mais utilizados são butil-hidroxianisol (BHA), butilhidroxitolueno (BHT) e terc-butil-hidroquinona (TBHQ). Ainda nesse contexto, a curcumina, um antioxidante de origem natural, juntamente com seus derivados sintéticos têm sido sugeridos para uso como suplemento alimentar, atuando como protetores do organismo contra o estresse oxidativo. (GUTIERRES et al., 2012; DU et al., 2013; PALMA et al., 2014; CHUENGSAMARN et al., 2014).

A curcumina é um pigmento de tom amarelado a alaranjado extraído dos rizomas da Curcuma longa, uma espécie herbácea e perene da família Zingiberaceae, a mesma do gengibre. O nome do gênero é originário da palavra árabe kurkum que significa açafrão, com mais de 70 espécies já identificadas. Originária do sudeste asiático, essa planta foi introduzida no mundo ocidental no século XIV por exploradores europeus, ficando conhecida internacionalmente como turmeric, além de possuir diversos nomes populares como açafrão, falso-açafrão, açafrão-daÍndia e gengibre amarelo. (SHISHODIA et al., 2007; HATCHER et al., 2008; AGGARWAL; HARIKUMAR, 2009; VOLP et al., 2009; PIRES et al., 2013).

A cúrcuma, componente da dieta mediterrânea mundialmente conhecida pelos seus aspectos nutricionais positivos, é muito cultivada no sul e sudeste asiático, sendo a Índia considerada o principal país produtor, consumidor e exportador. No Brasil, tal espécie se adaptou bem, cultivada principalmente nos estados de Goiás, Mato Grosso e São Paulo, possuindo desenvolvimento altamente satisfatório e produtividade semelhante à de sua região de origem (CECILIO FILHO et al., 2000; CECILIO FILHO et al., 2004; SHISHODIA et al., 2007).

O rizoma da planta é amplamente utilizado na culinária como pigmento e tempero em molhos (curry), produtos de padaria, bolos, tortas, queijo, sorvete, iogurte, picles, mostarda, bebidas, sopas, cereais e coberturas de doces (SHISHODIA et al., 2007; VOLP et al., 2009). É também usada pela indústria de cosméticos, e na forma de cápsulas ou tabletes pela indústria farmacêutica pela sua tradição na medicina chinesa e indiana relacionada aos efeitos benéficos 
em várias doenças. Seu uso popular tem sido relacionado à prevenção e cura de diversos problemas de saúde como distúrbios biliares, anorexia, tosse, queimaduras, acne, gases, reumatismo, sinusite, infecções comuns do olho, icterícia, várias doenças de pele, doenças hepáticas e feridas diabéticas, além da melhoria do bem-estar geral (CECILIO FILHO et al., 2000; WEBER et al., 2005, SHISHODIA et al., 2007; HATCHER et al., 2008; VOLP et al., 2009; PRASAD et al., 2014).

A cúrcuma possui cerca de 235 compostos cujo principal constituinte é a curcumina, com concentração no rizoma seco da planta variando de 2 a 5\%. (PEREIRA; MOREIRA, 2009; AGGARWAL et al., 2013). Isolada pela primeira vez em 1815 e obtida na forma cristalina em 1870, a curcumina vem sendo amplamente estudada e se destaca por possuir grande poder antioxidante, agindo assim na proteção de biomoléculas ao diminuir efeitos oxidativos ou aumentar a ação de enzimas antioxidantes. Além disso, também são bem estabelecidas suas atividades anti-inflamatória, antiparasitária, antifúngica, anticarcinogênica, hepato e nefroprotetora, supressora de trombose, hipoglicêmica e antirreumática, existindo assim muitos dados na literatura que reportam grande variedade de atividades farmacológicas por meio de diferentes mecanismos (ANAND et al., 2007; ANAND et al., 2008; VOLP et al., 2009; GUTIERRES et al., 2012).

Quimicamente, a curcumina é o principal representante dos pigmentos curcuminóides, que pertencem à classe diferuloilmetano $\left(\mathrm{C}_{21} \mathrm{H}_{20} \mathrm{O}_{6}\right)$. Estruturalmente, é caracterizada como uma molécula de dibenzoil-metano (1,7-bis-(hidroxi-3-metoxifenil)-1,6-heptadieno-3,5-diona), exibindo tautomerismo ceto-enólico com predominância da forma ceto em meio neutro e ácido e da forma enol em meio alcalino. (ANAND et al., 2007; SINGH et al., 2011).

O grande potencial antioxidante e anti-inflamatório da curcumina faz com que esta seja candidata a estudos para terapia no diabetes por sequestrar ERs em situações de estresse oxidativo e por impedir a peroxidação lipídica, atuando, portanto, na proteção de biomoléculas. (GUTIERRES et al., 2012; PALMA et al., 2014). Acredita-se que as reações com ERs são iniciadas por ambas hidroxilas fenólica e enólica. No organismo, a curcumina possui vários alvos, ligando-se a metais, albumina e outras moléculas, possibilitando inibição da atividade de enzimas, do crescimento tumoral, da angiogênese, da ativação de fatores de transcrição que regulam a expressão de genes associados ao surgimento de tumores, da inflamação, entre outros. (SINGH et al., 2011; SOETIKNO et al., 2012; MENG et al., 2013; PRASAD et al., 2014).

Os efeitos potencialmente benéficos dessa substância foram verificados em vários estudos utilizando modelos de animais diabéticos. O uso da curcumina como tratamento ou parte 
dele, em diferentes formas de administração, seja incorporada ao alimento, solubilizada em extratos etanólicos, suspendida em soluções de carboximetilcelulose (CMC) ou mesmo em água, apresentou melhoria em diversos parâmetros relacionados ao DM, como redução nos níveis séricos de glicose, hemoglobina glicada, colesterol e triacilgliceróis, proteção das ilhotas pancreáticas, diminuição da lipoproteína de baixa densidade (LDL) e aumento da de alta densidade (HDL), redução da hiperlipidemia, diminuição da atividade sérica das enzimas AST (aspartato aminotransferase) e ALT (alanina aminotransferase), aumento de peso corporal, melhora nas lesões renais, retardo no desenvolvimento de catarata, aumento dos níveis de insulina, redução dos níveis de espécies reativas ao ácido tiobarbitúrico (TBARS) no soro, fígado e rins, prevenção da redução nos níveis de enzimas antioxidantes, entre outros. (GUTIERRES et al., 2012; MENG et al., 2013; CHUENGSAMARN et al., 2014; PALMA et al., 2014).

Adicionalmente, a molécula de curcumina possui um excelente perfil de segurança em animais e humanos, sem apresentar toxicidade mesmo em altas doses (superiores a 100 $\mathrm{mg} / \mathrm{kg} / \mathrm{dia}$ ), o que associado às suas propriedades farmacológicas a torna uma potencial substância para o auxílio no tratamento de uma variedade de doenças, como por exemplo, o diabetes mellitus, hipertensão arterial, osteoporose, Alzheimer e Parkinson. Por outro lado, apesar de sua eficácia e segurança, tal substância não se mostra um bom agente terapêutico. Sua baixa solubilidade aquosa, baixa biodisponibilidade (baixa absorção, rápido metabolismo e rápida eliminação sistêmica), e cor intensa foram apontadas como os principais problemas que impedem que suas propriedades funcionais sejam plenamente exploradas (ANAND et al., 2007; DU et al., 2013; MENG et al., 2013; SHELMA; SHARMA, 2013; PRASAD et al., 2014).

Devido a esses fatos, várias estratégias têm sido avaliadas para a obtenção de análogos da curcumina apresentando eficácia semelhante ou superior e propriedades físico-químicas mais adequadas. A estrutura química da curcumina é determinante para sua atividade biológica e modificações estruturais vêm demonstrando, in vitro e in vivo, uma possibilidade de modulação de sua biodisponibilidade, com o intuito de elevar seus efeitos biológicos benéficos (SHISHODIA et al., 2005; ANAND et al., 2007; ANAND et al., 2008).

Nesse contexto, vários análogos estruturais da curcumina têm sido sintetizados com intuito de elucidar porções essenciais da molécula para a atividade biológica (grupos farmacofóricos), sendo encontrados análogos com maior e menor atividade biológica quando comparados à curcumina (VENKATESAN; RAO, 2000; RUKKUMANI et al., 2004; YOUSSEF et al., 2004; SELVAM et al., 2005; WEBER et al. 2005; CHANDRU et al., 2007; TAMVAKOPOULOS et al., 2007; TOMREN et al., 2007; SUBRAMANIAM et al., 2008; DAI 
et al., 2009; LEE et al., 2009; ERYANTI et al., 2011; SHANG et al., 2010; SINGH et al., 2011; BHULLAR et al., 2013; DU et al., 2013; LI et al., 2013).

Muitos estudos já revelam algumas relações estrutura-atividade, por exemplo, a presença dos grupos fenólicos é importante para a atividade biológica e antioxidante e a introdução de grupos metoxila $\left(-\mathrm{OCH}_{3}\right)$ nos anéis aromáticos pode otimizar a atividade. (VENKATESAN; RAO, 2000; TOMREN et al., 2007; SINGH et al., 2011). Outros estudos mostraram ainda que o grupo metileno $\left(-\mathrm{CH}_{2}-\right)$ central da curcumina é importante para sua atividade antioxidante. (WEBER et al., 2005; SHANG et al., 2010).

Portanto, a origem da curcumina a partir de fontes naturais, sua baixa toxicidade, estrutura relativamente simples e vasta gama de propriedades farmacêuticas a torna uma candidata promissora para uso medicinal, representando um protótipo ideal para modificações e otimizações químicas na busca por novos suplementos alimentares (BHULLAR et al., 2013; DU et al., 2013).

\section{Considerações Finais}

Com o aumento da incidência de doenças crônico-degenerativas como DM, tem crescido o interesse e a necessidade pela busca de substâncias antioxidantes capazes de prevenir o agravamento ou o surgimento de tais doenças. Essa busca tem sido direcionada para substâncias de origem natural e o posterior desenvolvimento de análogos a partir delas, visando melhorar suas características. Nesse contexto, a curcumina é uma molécula muito propícia, devido à suas características e propriedades, que a tornam, bem como a seus análogos, ótimos candidatos a serem utilizados como suplementos alimentares em uma alimentação funcional, visando proteger o corpo contra os danos gerados pelo estresse oxidativo. Assim, com a busca por suplementos alimentares mais promissores torna necessária a regularização dos suplementos dessa natureza para garantir ao consumidor segurança e eficácia das alegações funcionais com base em avaliações científicas e no histórico de uso dos produtos.

\section{Referências}

AGGARWAL, B.B.; HARIKUMAR, K.B. Potential therapeutic effects of curcumin, the antiinflammatory agent, against neurodegenerative, cardiovascular, pulmonary, metabolic, autoimmune and neoplastic diseases. The International Journal of Biochemistry \& Cell Biology, v. 41, n. 1, p. 40-59, 2009.

AGGARWAL B.B., YUAN W., LI S., GUPTA S.C. Curcumin-free turmeric exhibits antiinflammatory and anticancer activities: Identification of novel components of turmeric. Molecular Nutrition \& Food Research. v.57, p.1529-1542, 2013. 
AK, T.; GÜLÇIN, I. Antioxidant and radical scavenging properties of curcumin. ChemicoBiological Interactions, v. 174, p. 27-37, 2008.

ANAND, P.; KUNNUMAKKARA A.B.; NEWMAN R.A.; AGGARWAL B.B. Bioavailability of curcumin: problems and promises. Molecular Pharmaceutics, v. 4, n. 6, p. 807-818, 2007.

ANAND, P.; THOMAS S.G.; KUNNUMAKKARA A.B.; SUNDARAM C.; HARIKUMAR K.B.; SUNG B. Biological activities of curcumin and its analogues (Congeners) made by man and Mother Nature. Biochemical Pharmacology, v. 76, p. 1590-1611, 2008.

ANVISA. Brasília: Portaria $\mathrm{n}^{\circ}$ 32, de 13 de janeiro de 1998. - Diário Oficial da União;Disponível em:

$<$ http://portal.anvisa.gov.br/wps/wcm/connect/23b38c80400ce15aa869ee6d6e8afaaa/Portaria+n $\% \mathrm{C} 2 \% \mathrm{BA}+32-1998+\% 28$ vers\%C3\%A3o+DOU+15-01-98\%29.pdf?MOD=AJPERES>. Acesso em: 30 abril. 2018.

ARORA, S.; OJHA, S. K.; VOHORA, D. Characterisation of streptozotocin induceddiabetes mellitus in Swiss albino mice. Global Journal of Pharmacology, v. 3, n. 2, p. 81-84, 2009.

BARBOSA, K.B.F.; COSTA N.M.B.; ALFENAS R.C.G.; PAULA S.O.; MININ V.P.R.; BRESSAN J. Estresse oxidativo: avaliação de marcadores. Nutrire: Revista da Sociedade Brasileira de Alimentação e Nutrição, v. 33, n. 2, p. 111-128, 2008.

BARBOSA, K.B.F. COSTA N.M.B.; ALFENAS R.C.G.; PAULA S.O.; MININ V.P.R.; BRESSAN J. Estresse oxidativo: conceito, implicações e fatores modulatórios. Revista de Nutrição, v. 23, n. 4, p. 629-643, 2010.

BHULLAR, K. S.; JHA A.; YOUSSEF D.; RUPASINGHE H.P.V. Curcumin and its carbocyclic analogs: structure-activity in relation to antioxidant and selected biological properties. Molecules, v. 18, p. 5389-5404, 2013.

CAROCHO, M.; FERREIRA, I.C.F.R. A review on antioxidants, prooxidants and related controversy: Natural and synthetic compounds, screening and analysis methodologies and future perspectives. Food and Chemical Toxicology, v. 51, p. 15-25, 2013.

CHAMPE, P.C.; HARVEY R.A.; FERRIER D.R. Bioquímica ilustrada. 3 ed. Porto Alegre: Ed. Artmed, p. 335-346, 2006.

CHANDRU, H.; SHARADA A.C.; BETTADAIAH B.K.; KUMAR C.S.A.; RANGAPPA K.S.; SUNILA J.K. In vivo growth inhibitory and anti-angiogenic effects of synthetic novel dienonecyclopropoxycurcumin analogs on mouse Ehrlich ascites tumor. Bioorganic \& Medicinal Chemistry, v. 15, p. 7696-7703, 2007.

CHOI, H. Antioxidant activity of Curcuma Longa L., novel foodstuff. Molecular \& Cellular Toxicology, v. 5, n. 3, p. 237-242, 2009.

CHUENGSAMARN, S.; RATTANAMONGKOLGUL $\quad$ S.; $\quad$ PHONRAT $\quad$ B.; TUNGTRONGCHITR R.; JIRAWATNOTAI S. Reduction of atherogenic risk in patients with type 2 diabetes by curcuminoid extract: a randomized controlled trial. Journal of Nutritional Biochemistry, v. 25, p. 144-150, 2014.

DAI, F.; CHENG W.F.; ZHOU B.; YANG L.; LIU Z.L. Antioxidative effects of curcumin and its analogues against the free-radical-induced peroxidation of linoleic acid in micelles. Phytotherapy Research, v. 23, p. 1220-1228, 2009.

DEVCICH, D. A.; PEDERSEN, I. K.; PETRIE, K. J. You eat what you are: Modern health worries and the acceptance of natural and synthetic additives is functional foods. Appetite, v. 48, p. 333-337, 2007. 
DIABETES. Brasil: Sociedade brasileira de diabetes. Disponível em: <http://www.diabetes.org.br/>. Acesso em: 30 abril 2018.

DU, Z.; WEI X.; HUANG M.T.; ZHENG X.; LIU Y.; CONNEY A.H. Anti-proliferative, antiinflammatory and antioxidant effects of curcumin analogue $A_{2}$. Archives of Pharmacal Research, v. 36, n. 10, p. 1204-1210, 2013.

ERYANTI, Y.; NURULITA, Y.; HENDRA, R.; YUHARMEN, SYAHRI J.; ZAMRI A. Synthesizing derivatives from cyclopentanone analogue curcumin and their toxic, antioxidant and anti-inflammatory activities. MakaraJournalof Science, v. 15, n. 2, p. 117-123, 2011.

FERREIRA, A. L. A.; MATSUBARA, L. S. Radicais livres: conceitos, doenças relacionadas, sistema de defesa e estresse oxidativo. Revista da Associação Médica Brasileira, v. 43, n. 1, p. 61-68, 1997.

FILHO CECÍLIO, A. B.; SOUZA R.J.; BRAZ L.T.; TAVARES M. Cúrcuma: planta medicinal, condimentar e de outros usos potenciais. Ciência Rural, v. 30, n. 1, p. 171-175, 2000.

FILHO CECÍLIO, A. B.; SOUZA R.J.; FAQUIN V.; CARVALHO C.M. Época e densidade de plantio na produção de cúrcuma. Ciência Rural, v. 34, n. 4, p. 1021-1026, 2004.

GARROW, D.; EGEDE, L. E. Association between complementary and alternative medicine use, preventive care practices, and use of conventional medical services among adults with diabetes. Diabetes Care, v. 29, n. 1, p. 15-19, 2006.

GROTTO, D.; MARIA L.S.; VALENTINI J.; PANIZ C.; SCHMITT G.; GARCIA C. Importance of the lipid peroxidation biomarkers and methodological aspects for malondialdehyde quantification. Química Nova, v. 32, n. 1, p. 169-174, 2009.

GUIA alimentar para a população brasileira, 2014. Disponível em: <http://189.28.128.100/dab/docs/portaldab/publicacoes/guia_alimentar_populacao_brasileira.pdf >. Acesso em: 30 abril 2018.

GUTIERRES, V. O.; PINHEIRO C.M.; ASSIS R.P.; VENDRAMINI R.C.; PEPATO M.T.; BRUNETTI I.L. Curcumin-supplemented yoghurt improves physiological and biochemicalmarkers of experimental diabetes. British Journal of Nutrition, v. 108, p. 440-448, 2012.

HALLIWELL, B.; CHIRICO, S. Lipid peroxidation: its mechanism, measurement and significance. The American Journal Clinical, v. 57, p. 715S-725S, 1993.

HATCHER, H.; PLANALP R.; CHO J.; TORTI F.M.; TORTI S.V. Curcumin: From ancient medicine to current clinical trials. Cellular and Molecular Life Sciences, n. 65, p. 1631-1652, 2008 .

HENRIQUES, G. S.; LOPES C.V.A.; SIMEONE M.L.F.; BINDA C.C. Aplicação do método de índice glicêmico em modelo de ratos: padronização utilizando fórmula enteral de composição definida para animais normais e feitos diabéticos por aplicação de estreptozotocina. Revista do Médico Residente, v. 12, n. 2, p. 67-78, 2010.

HONORATO, T. C.; BATISTA E.; NASCIMENTO K.O.; PIRES T. Aditivos alimentares: aplicações e toxicologia. Revista Verde, v. 8, n. 5, p. 01-11, 2013.

HOROWITZ, C. R.; COLSON K.A.; HEBERT P.L.; LANCASTER k. Barriers to buying healthy foods for people with diabetes: evidence of environmental disparities. American Journal of Public Health, v. 94, n. 9, p. 1549-1554, 2004.

HUSSEIN, H. K.; ABU-ZINADAH, O. A. Antioxidant effect of curcumin extracts in induced diabetic Wister rats. International Journal of Zoological Research, 2010. 
JEENGER, M. K.; SHRIVASTAVA S.; YERRA V.G.; NAIDU V.G.M.; RAMAKRISHNA S.; KUMAR A. Curcumin: A pleiotropic phytonutrient in diabetic complications. Nutrition, p. 1-8, 2014. Disponível em: 〈http://dx.doi.org/10.1016/j.nut.2014.06.015>. Acesso em: 30 abril 2018.

KOHEN, R.; NYSKA, A. Oxidation of biological systems: oxidative stress phenomena, antioxidants, redox reactions, and methods for their quantification. Toxicologic Pathology, v. 30, n. 6, p. 620-250, 2002.

KUMAR, T. P.; ANTONY S.; SOMAN S.; KURUVILLA K.P.; GEORGE N.; PAULOSE C.S. Role of curcumin in the prevention of cholinergic mediated cortical dysfunctions in streptozotocin-induced diabetic rats. Molecular and Cellular Endocrinology, v. 331, p. 1-10, 2011.

LEE, K.; AZIZ F.H.A.; SYAHIDA A.; ABAS F.; SHAARI K.; ISRAF D.A. Synthesis and biological evaluation of curcumin-like diarylpentanoid analogues for anti-inflammatory, antioxidant and anti-tyrosinase activities. European Journal of Medicinal Chemistry, v. 44, p. 3195-3200, 2009.

LI, Y.; Zou X.; Cao K.; Xu J.; Yue T.; Dai F. Curcumin analog 1,5-bis(2-trifluoromethylphenyl)1,4-pentadien-3-one exhibits enhanced ability on Nrf2 activation and protection against acroleininduced ARPE-19 cell toxicity. Toxicology and Applied Pharmacology, v. 272, n. 3, p. 726-735, 2013.

LÓPEZ-ALARCÓN, C.; DENICOLA, A. Evaluating the antioxidant capacity of natural products: A review on chemical and cellular-based assays. Analytica Chimica Acta, v. 736, p. 1$10,2013$.

MAGALHÃES, L. M.; SEGUNDO M.A.; REIS S.; LIMA J.L.F.C. Methodological aspects about in vitro evaluation of antioxidant properties. Analytica Chimica Acta, v. 613, p. 1-19, 2008 .

MENG, B.; LI, J.; CAO, H. Antioxidant and Antiinflammatory Activities of Curcumin on Diabetes Mellitus and its Complications. Current Pharmaceutical Design, v. 19, p. 2101-2113, 2013.

NAJAFIAN, M. Effect of Curcumin on Metabolism of Lipids in Streptozotocinindiced Diabetic and normal Rats. Advances in Environmental Biology, v. 8, n. 12, p. 170-177, 2014.

NIKI, E. Assessment of Antioxidant Capacity in vitro and in vivo. Free Radical Biology \& Medicine, v. 49, p. 503-515, 2010.

ODEGARD, P. S.; JANCI, J. Prevalence and correlates of dietary supplement use in individuals with diabetes mellitus at an academic diabetes care clinic. The Diabetes Educator, v. 37, n. 3, p. 419-425, 2011.

PAHO, Pan American Health Organization. Disponível em: $<$ http://www.paho.org/bra/index.php?option=com_content $\&$ view $=$ article \&id=394:diabetesmellitus\&Itemid=539>. Acesso em: 30 abril 2018.

PALMA, H. E.; WOLKMER P.; GALLIO M.; CORRÊA M.M.B.; SCHMATZ R.; THOMÉ G.R. Oxidative stress parameters in blood, liver, and kidney of diabetic rats treated with curcumin and/or insulin. Molecular and Cellular Biochemistry, v. 368, p. 199-210, 2014.

PEEYUSH, K. T.; GIREESH G.; JOBIN M.; PAULOSE C.S. Neuroprotective role of curcumin in the cerebellum of streptozotocin-induced diabetic rats. Life Sciences, v. 85, p. 704-710, 2009.

PEREIRA, R. C. A; MOREIRA, M. R. Comunicado técnico 142: Cultivo de Curcuma longa L. (Açafrão-da-índia ou Cúrcuma). Embrapa, Fortaleza, Ceará, 2009. Disponível em: 

em: 30 abril 2018.

PIRES, L. F.; FREITAS, R. M.; RODRIGUES, A. C. T. Efeito antidepressivo e atividade serotoninérgica da curcumina em modelos de animais de depressão. Revista de Farmacologia de Chile, v. 6, n. 1, p. 37-44, 2013.

PRABHAKAR, P. K.; PRASAD R.; ALI S.; DOBLE M. Synergistic interaction of ferulic acid with commercial hypoglycemic drugs in streptozotocin induced diabetic rats. Phytomedicine, $\mathrm{v}$. 20, p. 488-494, 2013.

PRASAD, S.; TYAGI, A. K.; AGGARWAL, B. B. Recent Developments in Delivery, Bioavailability, Absorption and Metabolism of Curcumin: the Golden Pigment from Golden Spice. Cancer Research and Treatment, v. 46, n. 1, p. 2-18, 2014.

RECINE, E.; NASCIMENTO, M. A. B.; ITO, M. K. Guia Alimentar para pessoas com diabetes tipo 2. 2001. Disponível em: 〈http://189.28.128.100/nutricao/docs/geral/guia_diabetes.pdf $>$. Acesso em: 30 abril 2018.

REDDY, B. V.; SUNDARI J.S.; BALAMURUGAN E.; MENON V.P. Antihyperlipidemic effect of bis-1,7-(2-hydroxyphenyl)-hepta-1,6-diene-3,5-dione, a curcumin analog, on nicotine and streptozotocinteated rats. Molecular and Cellular Biochemistry, v. 335, p. 249-254, 2010.

RUKKUMANI, R.; ARUNA K.; VARMA P.S.; RAJASEKARAN K.N.; MENON V.P. Comparative effects of curcumin and an analog of curcumin on alcohol and PUFA induced oxidative stress. Journal of Pharmacy \& Pharmaceutical Sciences, v. 7, n. 2, p. 274-283, 2004.

SELVAM, C.; JACHAK S.M.; THILAGAVATHI R.; CHAKRABORTI A.K. Design, synthesis, biological evaluation and molecular docking of curcumin analogues as antioxidant, cyclooxygenase inhibitory and anti-inflammatory agents. Bioorganic \& Medicinal Chemistry Letters, v. 15, p. 1793-1797, 2005.

SENADO. Brasília: Projeto de lei do senado $\mathrm{n}^{\circ}$ 233, de 2014. Disponível em: $<$ http://www.senado.gov.br/atividade/materia/getPDF.asp?t=153032\&>. Acesso em: 30 abril 2018 .

SHANE-MCWHORTER, L. Dietary supplements and probiotics for diabetes. American Journal of Nursing, v. 112, n. 7, p. 47-53, 2012.

SHANG, Y.; JIN X.L.; SHANG X.L.; TANG J.J.; LIU G.Y.; DAI F. Antioxidant capacity of curcumin-directed analogues: Structure-activity relationship and influence of microenvironment. Food Chemistry, v. 119, p. 1435-1442, 2010.

SHARMA, J.; SINGH A.; SHARMA R.; SINGH Y.; TEOTIA U.V.S. Synthesis and antioxidante appraisal of curcumin and twocurcuminoid compounds. Der Pharmacia Sinica, v. 4, n. 3 , p. $151-163,2013$.

SHELMA, R.; SHARMA, C. P. In vitroand in vivo evaluation of curcumin loaded lauroyl sulphated chitosan for enhancing oral bioavailability. Carbohydrate Polymers, v. 95, n. 1, p. 441 448, 2013.

SHISHODIA, S.; CHATURVEDI, M. M.; AGGARWAL, B. B. Role of curcumin in cancer therapy. Current Problems in Cancer, v. 31, n. 4, p. 243-305, 2007. Disponível em: <http://cdn.elsevier.com/assets/pdf_file/0006/115719/current-problems-in-cancer-article-1.pdf $>$. Acessoem: 12 dez. 2015.

SHISHODIA, S.; SETHI, G.; AGGARWAL, B. B. Curcumin: getting back to the roots. Annals of the New York Academy of Sciences, v. 1056, p. 206-217, 2005. 
SILVA, L. F. M.; FERREIRA, K. S. Segurança alimentar de suplementos comercializados no Brasil. Revista Brasileira de Medicina do Esporte, v. 20, n. 5, p. 374-378, 2014.

SILVA, M.; LIMA W.G.; SILVA M.E.; PEDROSA M.L. Efeito da estreptozotocina sobre os perfis glicêmico e lipídico e o estresse oxidativo em hamsters. Arquivos Brasileiros de Endocrinologia \& Metabologia, n. 55, v. 1, p. 46-53, 2011.

SINGH, U.; BARIK A.; SINGH B.G.; PRIYADARSINI K.I. Reactions of reactive oxygen species (ROS) with curcumin analogues: Structure-activity relationship. Free Radical Research, v. 45, n. 3, p. 317-325, 2011.

SOETIKNO, V.; SARI F.R.; SUKUMARAN V.; LAKSHMANAN A.P; MITO S.; HARIMA M. Curcumin prevents diabetic cardiomyopathy in streptozotocin-induced diabetic rats: Possible involvement of PKC-MAPK signaling pathway. European Journal of Pharmaceutical Sciences, v. 47, p. 604-614, 2012.

SOETIKNO, V.; SARI F.R.; SUKUMARAN V.; LAKSHMANAN A.P.; HARIMA M.; SUZUKI K. Curcumin decreases renal triglyceride accumulation through AMPK-SREBP signaling pathway in streptozotocin-induced type 1 diabetic rats. Journal of Nutritional Biochemistry, v. 24, p. 796-802, 2013.

SUBRAMANIAM, D.; MAY R.; SUREBAN S.M.; LEE K.B.; GEORGE R.; KUPPUSAMY P. DiphenylDifluoroketone: A Curcumin Derivative with Potent In vivo Anticancer Activity. Cancer Research, v. 68, p. 1962-1969, 2008.

TAMVAKOPOULOS, C. DIMAS T.K.; SOFIANOS Z.D.; HATZIANTONIOU S.; HAN Z.; LIU Z.L. Metabolism and Anticancer Activity of the Curcumin analogue, Dimethoxycurcumin. Clinical Cancer Research, v. 13, p. 1269-1277, 2007.

TOMREN, M. A.; MÁSSON M.; LOFTSSON T.; TONNESEN H.H. Studies on curcumin and curcuminoids XXXI. Symemetric and asymmetric curcuminoids: Stability, activity and complexation with cyclodextrin. International Journal of Pharmaceutics, v. 338, p. 27-34, 2007.

VASCONCELOS, S.M.L.; GOULART M.O.F.; MOURA J.B.F.; MANFREDINI V.; BENFATO M.S.; KUBOTA L.T. Espécies reativas de oxigênio e de nitrogênio, antioxidantes e marcadores de danooxidativo em sangue humano: principais métodos analíticos para sua determinação. Química Nova, v. 30, n. 5, p. 1323-1338, 2007.

VENKATESAN, P.; RAO, M. N. A. Structure-Activity Relationships for the Inhibition of Lipid Peroxidation and the Scavenging of Free Radicals by Synthetic Symmetrical Curcumin Analogues. Journal of Pharmacy and Pharmacology, v. 52, p. 1123-1128, 2000.

VIEIRA, C.M.; CORDEIRO S.N.; JÚNIOR R.M.; TURATO E.R. Significados da dieta e mudanças de hábitos para portadores de doenças metabólicas crônicas: uma revisão. Ciência \& Saúde Coletiva, v. 16, n. 7, p. 3161-3168, 2011.

VOLP, A. C. P.; RENHE, I. R. T.; STRINGUETA, P. C. Pigmentosnaturaisbioativos. Alimentos e Nutrição, Araraquara, v. 20, n. 1, p. 157-166, 2009.

WEBER, W.M.; HUNSAKER L.A.; ABCOUWER S.F.; DECK L.M.; JAGT D.L.V. Antioxidant activities of curcumin and related enones. Bioorganic \& Medicinal Chemistry, v. 13, p. 3811-3820, 2005.

WHO, World Health Organization. Disponívelem: <http://www.who.int/diabetes/en/>. Acesso em: 30 abril 2018 .

YADAV, S.K.; SAH A.K.; JHA R.K.; SAH P.; SHAH D.K. Turmeric (curcumin) remedies gastroprotective action. Pharmacognosy Review, v. 7, n. 13, p. 42-46. 2013. 
YOUSSEF, K.M.; EL-SHERBENY M.A.; EL-SHAFIE F.S.; FARAG H.A.; AL-DEEB O.A.; AWADALLA S.A.A. Synthesis of Curcumin Analogues as Potential Antioxidant, Cancer Chemopreventive Agents. Arch. Pharm. Pharm. Med. Chem., v. 337, p. 42-54, 2004.

ZHAO, W.; ZHANG B.; LIAO M.J.; ZHANG W.X.; HE W.Y.; WANG H.B.; YANG C.X. Curcumin ameliorated diabetic neuropathy partially by inhibition of NADPH oxidase mediating oxidative stress in the spinal cord. Neuroscience Letters, v. 560, p. 81-85, 2014. 